\title{
Humanoid Dynamic Controller
}

\author{
Rui Monteiro', Luís Paulo Reis ${ }^{2,4}$, António Correia Pereira ${ }^{{ }^{3,4}}$ \\ ${ }^{1}$ University of Porto, Faculty of Engineering (FEUP) - Dept. of Electrical and Computer Eng. (DEEC) \\ Rua Dr. Roberto Frias, s/n, 4200-465 Porto, Portugal, +351 225081600 \\ ${ }^{2}$ University of Minho, School of Engineering (EEUM) - Information System Department (DSI) \\ Campus de Azurém, 4800-058 Guimarães, Portugal, +351 253510312 \\ ${ }^{3}$ University of Porto, Faculty of Engineering (FEUP) - Department of Informatics Engineering (DEI) \\ Rua Dr. Roberto Frias, s/n, 4200-465 Porto, Portugal, +351 225082134 \\ ${ }^{4}$ Artificial Intelligence and Computer Science Laboratory (LIACC), University of Porto (UP) \\ Rua Dr. Roberto Frias, s/n, 4200-465 Porto, Portugal, +351 220402900 \\ *corresponding author, e-mail: rui.edgar.monteiro@fe.up.pt, Ipreis@dsi.uminho.pt, amcp@fe.up.pt*
}

\begin{abstract}
In the last years several investigators have focused on the development and control of humanoid robots able to acquire human behaviors and features, in order to be able to interact with humans and work together with them. The fall of a humanoid robot can lead to damages, which entail costs for repair or even human health risks, so ensuring stability is a main concern in terms of cost and safety. This document presents the implementation of a dynamic controller for humanoid robots, able to position the robot's center of mass in a certain location operating as a stability module. It is also described a precise behavior of preparing an omnidirectional kick for a humanoid robot by placing the support foot in a precise position and orientation calculated in accordance with the robot's position, the ball and the target, and by transferring the robot's center of mass to the desired position.
\end{abstract}

Keywords: Humanoid Stability; Dynamic Controller; Prepare Kick.

Copyright $\odot 2012$ Universitas Ahmad Dahlan. All rights reserved.

\section{Introduction}

In the last two decades, the area of humanoid robotics has shown significant improvements. This evolution results from the development of new actuators, computers and other technologies that make possible the construction of a machine with human-like skills. Since 1960 the robotics industry has been growing and so has been the research in this area where several universities such as Waseda University [1] and Tokyo, and companies like Honda [2], Boston Dynamics and Aldebaran Robotics [3] have done research work.

The main purpose in the humanoid robots research is to allow the robots to have the ability to work together with humans due to their flexibility and adaptability that allow them to use human tools and human environments. However, it is necessary to ensure the safety of both human and robots.

The control theory is a branch of engineering with the aim of controlling a dynamic system through its inputs. The application of these controllers is extended to large areas, including robotics, where they are frequently used to control the servo motors and in stability modules. In engineering, a control system has at least two main modules, the controller itself and the system to be controlled. A stability module is essential for humanoid robot's stability using a dynamic controller to achieve the desired set points [4]. The proposed controller objective is to keep the robot statically stable, positioning the center of mass in a desired set point to ensure the robot's stability during precise movements.

The work described in this article was developed in the scope of the Portuguese soccer team FCPortugal, competing in the world's RoboCup 3D Simulation League and Standard Platform League [5-8], in order to reach a behavior combined with others previously developed [9-10] to equip a humanoid robot with the ability to kick a ball in various directions, perform a kick/pass without the preparation phase, and maintaining the humanoid body stability, creating a team of NAO robots [3], real or virtual, with the capability to play a soccer match decently. 


\section{The Center of Mass Dynamic Controller}

The developed controller allows the use of various controlling architectures to control the projection of the center of mass (CoM) on the ground through a PID controller. All the developed control methods were adjusted according to the Ziegler Nichols heuristic method [11].

\subsection{The arms}

Since the robot arms during a football match are rarely used, it was initially developed a controller that uses the robot arms to position the center of mass.

The proposed module uses a PID controller that receives the error position of the center of mass $(x, y)$ relative to the robot's shoulder and calculates the desired position for the robot arms $(x, y)$. As for purposes of stability, the position of the arm should be as close as possible to the support plane, the $\mathrm{z}$ coordinate of the arms is the lowest in the robot workspace and can be calculated from the length of the arm and the position $(x, y)$ with:

$$
\mathrm{z}=-\sqrt{\mathrm{l}_{\mathrm{arm}}^{2}+\mathrm{x}^{2}+\mathrm{y}^{2}}
$$

The coordinates obtained in equation 1 are then applied to the inverse kinematics module to place the robot arm in the desired position controlling the robot's center of mass. Applying the pose illustrated in Figure 1 the position of the center of mass of the robot has the response shown in Figure 2.

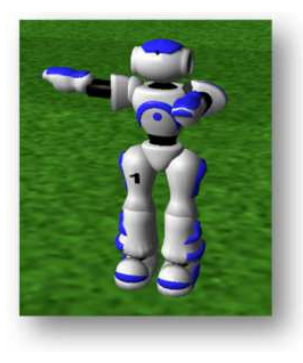

Figure 1. Projection of the robot arm along the $y$ axis
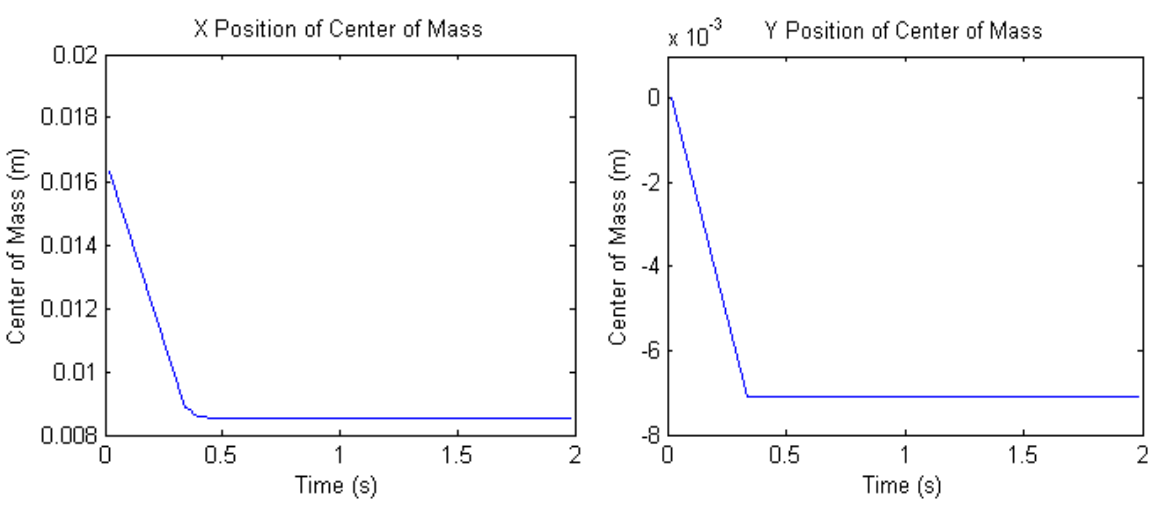

Figure 2. Robot's CoM response

This method has proved to be ineffective in controlling the position of the robot's center of mass because the robot arms mass is too small relative to its total mass [3] and therefore the center of mass manipulability with this method is very low. In Figure 2.2 it can be seen that the arm can only change the robot's center of mass position for about $9 \mathrm{~mm}$ in the xy plane.

\subsection{The legs}

To control robot's center of mass position, the robot's phase of movement must be taken into account. Figure 3 represents the phases of humanoid locomotion.

As can be seen, the phases "a" and "c" correspond to the double support phase, in which both feet are in contact with the ground, and the phases "b" and "d" correspond to single support phase in which only one foot is in contact with the ground.

\subsection{Double support phase}

In the double support phase pitch and roll of both foot and hip joints are used. Thus, using an angle between each pair of hip joints, and applying the opposite angle in the foot joints, the robot's body is moved parallel to the ground. This way we can control the $x$ position of the robot through the pitch angle applied to the robot's foot and hip joints, and we can control the $y$ position of the robot through the roll angle applied to the robot's foot and hip joints. This control method is shown in Figure 4. 


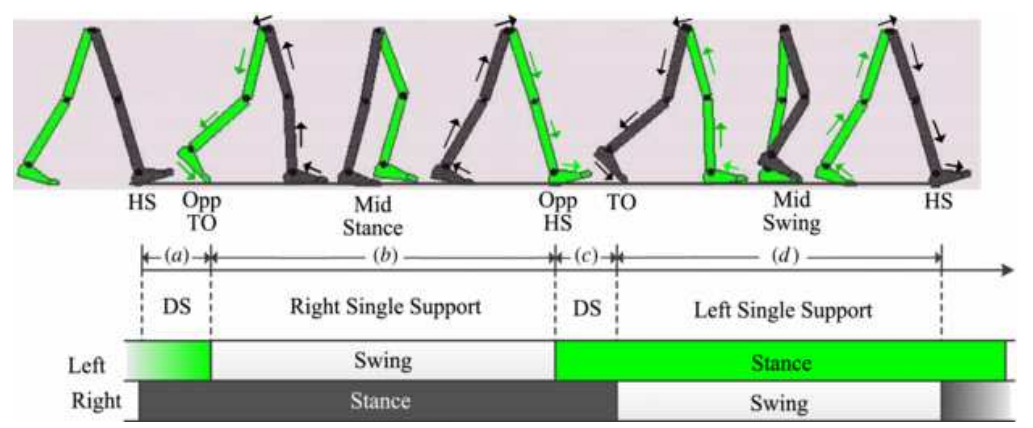

Figure 3. Phases of humanoid locomotion
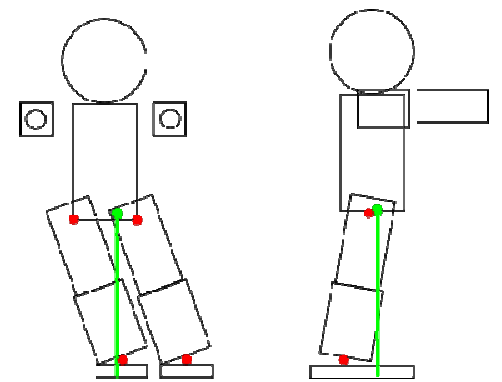

Figure 4. Controlling the robot's center of mass in double support phase

Figure 5 shows the response of the controller after the application of a square wave input ranging from the position $(0,0)$ to $(-0.05,-0.02)$, in meters, with a 6 seconds period. Analyzing the response we can verify that the system presents an under damped response with low rise and settling times. This analysis demonstrates that the controller is accurate and ideal for situations that require an exact positioning of the robot's center of mass.
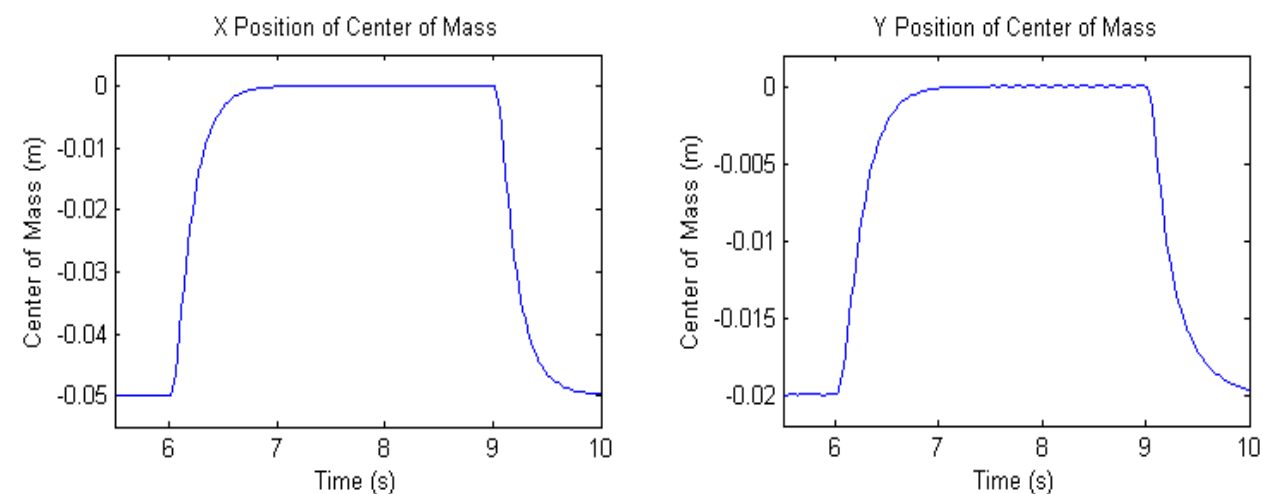

Figure 5. Position of the robot's center of mass being controlled in double support phase

\subsection{Single support phase}

In the single support phase we propose two different methods to control the robot's center of mass position. We can use only the hips pitch and roll joints, and then we can position the robot's center of mass by changing the robot's torso orientation, or we can use both hip and foot joints just like in double support phase.

As can be seen in Figure 6, by applying an angle determined by the PID controller to the robot's foot and hip joints, with the latter having opposite sign of the first, it is possible to move the robot's center of mass while maintaining the orientation of the trunk. 
Figure 7 shows the response of the controller after the application of an input square wave ranging from the position $(0,0)$ to $(-0.05,-0.02)$, in meters, with a 6 seconds period. Analyzing this figure we can verify that the system has an under damped response with low rise and settling times. This method's response is quite similar to the one presented by the double support phase due to their related features. This method is accurate and ideal for applications which require precise positioning of the robot's center of mass.
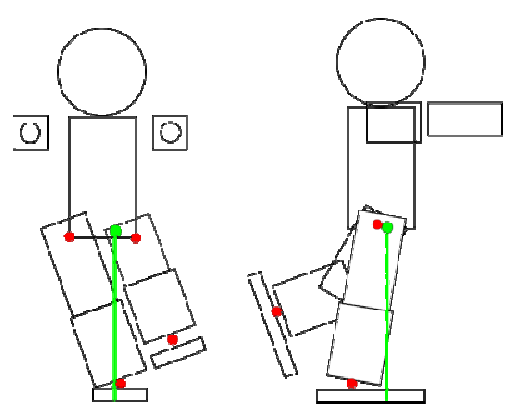

Figure 6. Controlling the robot's center of mass in single support phase with hip and foot joints.
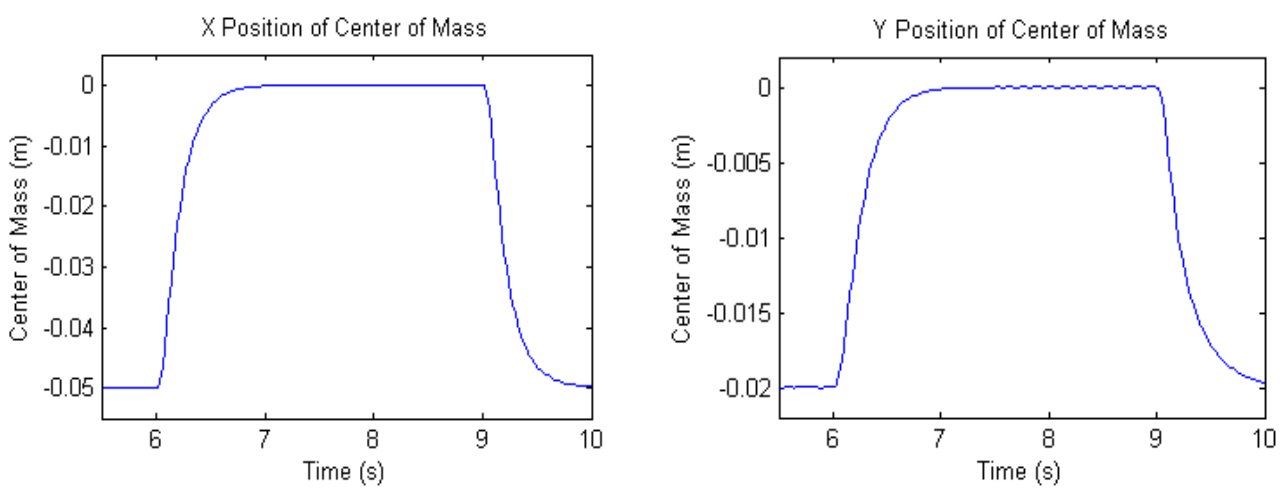

Figure 7. Position of the robot's center of mass being controlled in single support phase with foot and hip joints

The control sequence of the method mentioned above is shown in Figure 8.

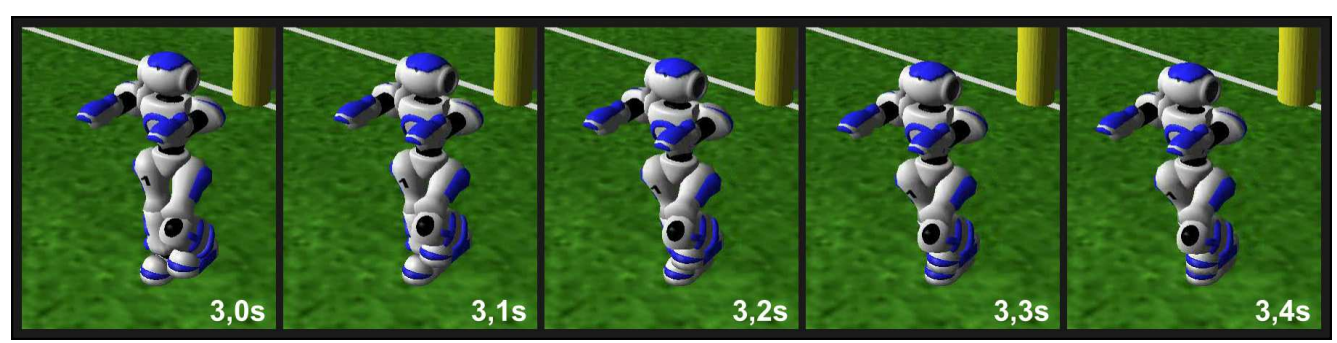

Figure 8. Control sequence of the robot's center of mass being controlled by the foot and hip joints in single support phase

As can be seen in Figure 9, by applying an angle determined by the PID controller to the robot's hip joints we can move the robot's center of mass. Thus, the robot remaining joints are available to perform any task required by the robot's behavior. Using only two joints, this method is the least invasive way to control the robot's center of mass. 
Figure 10 shows the response of the controller after the application of an input square wave ranging from the position $(0,0)$ to $(-0.05,-0.02)$, in meters, with a 6 seconds period. By analyzing figure it is possible to verify that the system presents an over damped response with minimal rise time and low overshoot of approximately $5 \%$. This control method is fast and precise being suitable for situations which require fast positioning of the robot's center of mass as in case of robots balance.
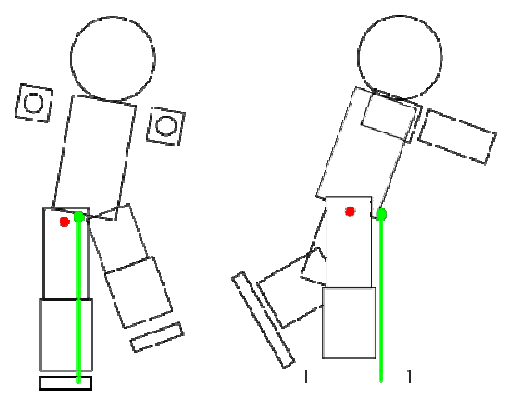

Figure 9. Controlling the robot's center of mass in single support phase with hip joints.
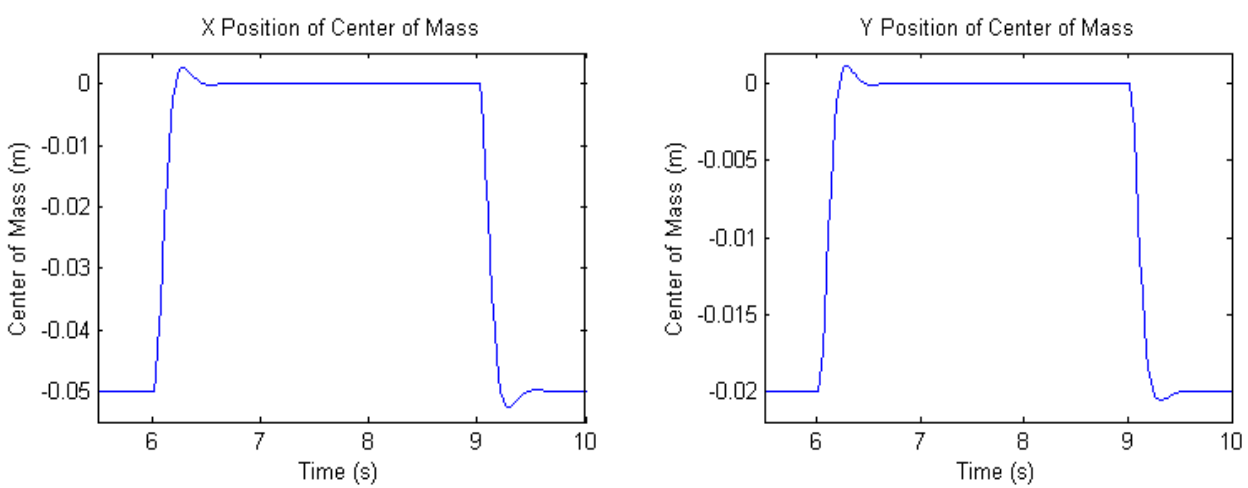

Figure 10. Position of the robot's center of mass being controlled in single support phase with hip joints

The control sequence of the method mentioned above is shown in Figure 11.

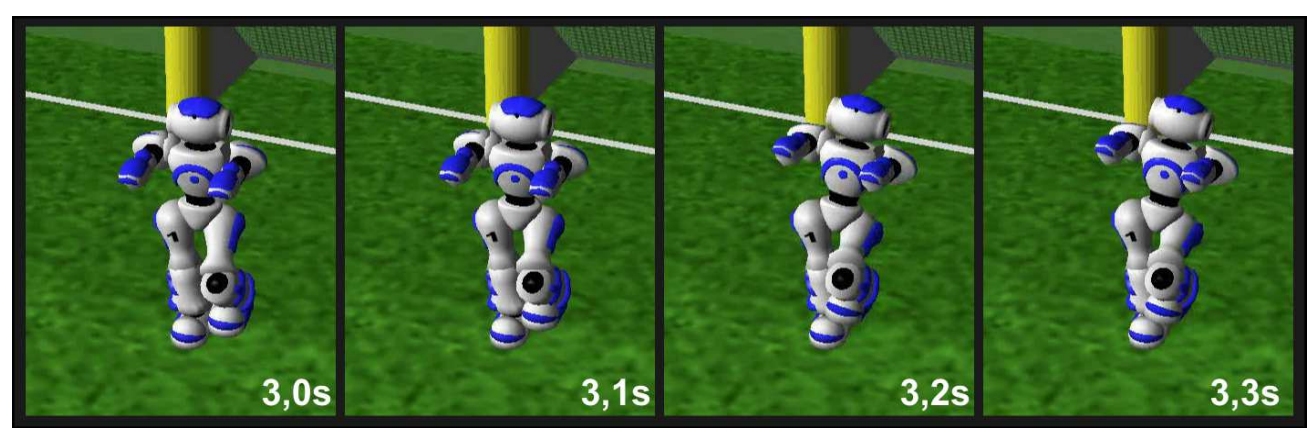

Figure 11. Control sequence of the robot's center of mass being controlled by the hip joints in single support phase

By overlaying the response of the method using hip and foot joints represented in Figure 7, with the response of the method using only hip joints represented in Figure 10, we obtained the graph presented in Figure 12. We can observe that we can obtain a faster response using the hip joints method than using both joints, but the first one has an overshoot of nearly $5 \%$. Thus, the hip and foot joints method should be used in situations which require the orientation of the trunk to remain constant during the movement or in applications where it 
isn't allowed overshoot, as in the precise positioning of the robot's center mass around the ends of the robot's support polygon. The method that only uses the hip joints should be used in all other situations and is especially suited for balance situations due to its fast response and the possibility of overshoot.
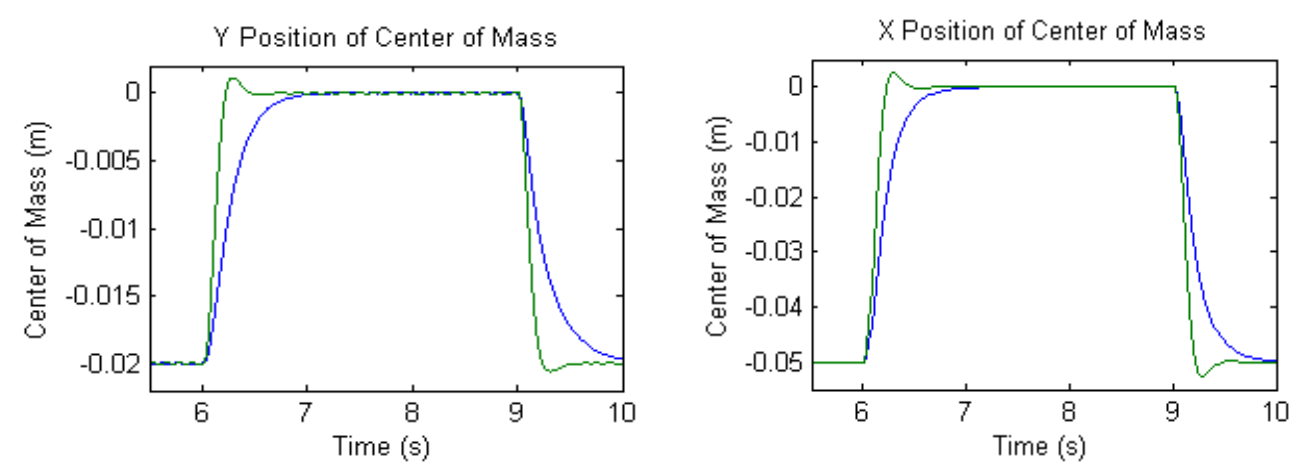

Figure 12. Comparison of response between the method that uses the hip and feet joints (blue) and the method that only uses the hip joints (green)

\section{The Prepare Kick}

\subsection{World analysis}

In order to allow a dynamic and flexible prepare kick movement it is necessary to perform a world analysis and then calculate the exact support foot position for the kick's execution. Therefore, it is necessary to consider three objects: player; ball and target.

Using these set of objects it is possible to calculate the foot position and orientation relative to the player using a set of parameters obtained by optimizing the best foot start position to the kick and relating the three objects mentioned. The parameters are:

- dist - Distance from the foot position to the ball;

- $\theta$ - Angle between the target and the foot position;

- $\quad \alpha$ - Foot orientation relative to the target.

Initially, the target and ball coordinates are corrected according to the robot's orientation so they can be expressed according to the robot's frame. The Figure 12 outlines the calculation of the position where the robot must place the support foot.

In this calculation is applied a rotation to the vector that goes from the target to the ball, in blue, of $\theta$ and its length is normalized to dist. Thus, the point where the robot must put his foot is obtained through the sum of the vector that goes from the robot to the ball with the vector that goes from the ball to the support point, in red.

The foot orientation angle relative to the robot's initial pose is given by the sum of the angle formed between the ball and the target, with the angle $\alpha$ given as a parameter.

\subsection{Motion planning}

The implemented movement can be decomposed in five phases, being: transition from double support to single support, positioning the support foot, lowering the body, transferring the robot's center of mass and raise phase. Among these, the phases of positioning the support foot and lowering the body are performed in parallel.

\subsubsection{Transfer to single support}

The strike movement preparation begins with the assumption that the robot is in a stable position in the phase of double support. This security can be obtained by analyzing the position of center of mass relative to the support polygon, as described in section 2 . In order to perform a step, it is necessary to place the robot only supported by one leg, and for that the first stage of preparation consists in transferring the shot from the center of mass of the robot to the contact surface between the robot foot that will shoot and soil, then lifting the opposite foot. 


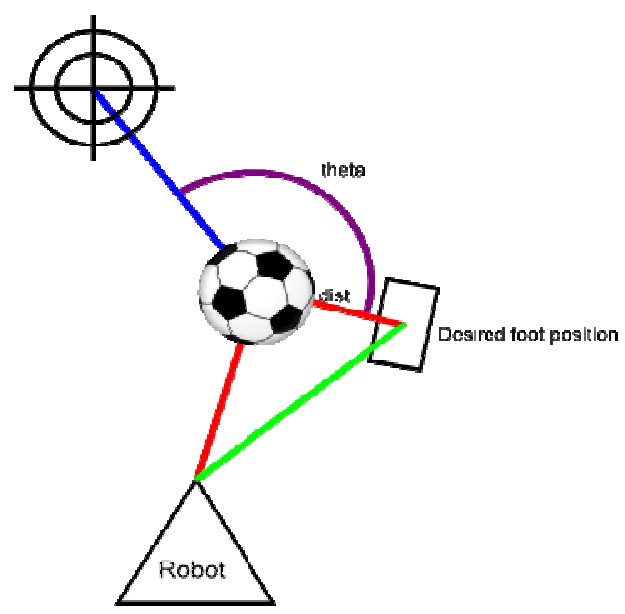

Figure 12. Calculation of the robot's support foot position

Figure 13 represents the robot transferring to single support.

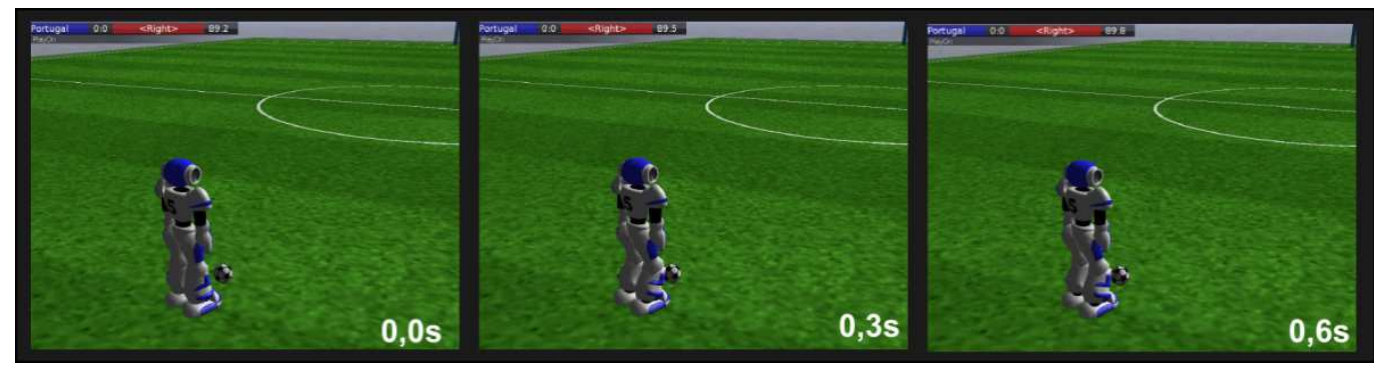

Figure 13. Following transfer of the robot to single support

\subsubsection{Support foot placing}

Since the workload of the leg of the robot, shown in Figure 14 by a semicircle in red, does not contain the end point of the foot support desired, represented in blue, the robot should set foot in the point whose projection in the semicircle $z$ intersects representative of the workload. Thus, by lowering the foot opposite the robot places the support foot in the desired position. Thus, the stand follows a Bezier curve of degree 3 that will take the walk to this position in a circular path. To put the kick foot in the desired position the robot will lower the body according to the elevation of the foot set in the previous paragraph and shown in Figure 15. The foot should follow a linear path down which ends when the robot returns to the position of double support.

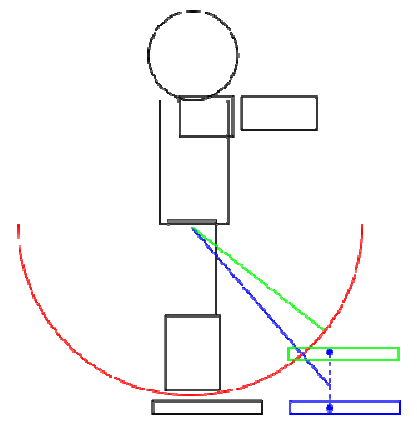

Figure 14. Robot's leg workload

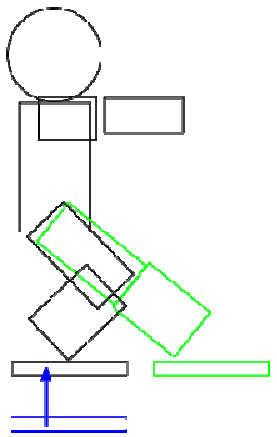

Figure 15. Robot's body lowering 


\subsubsection{Robot's body lowering}

The Figure 16 represents the parallel execution of the lowering of the robot body and placing the foot support.

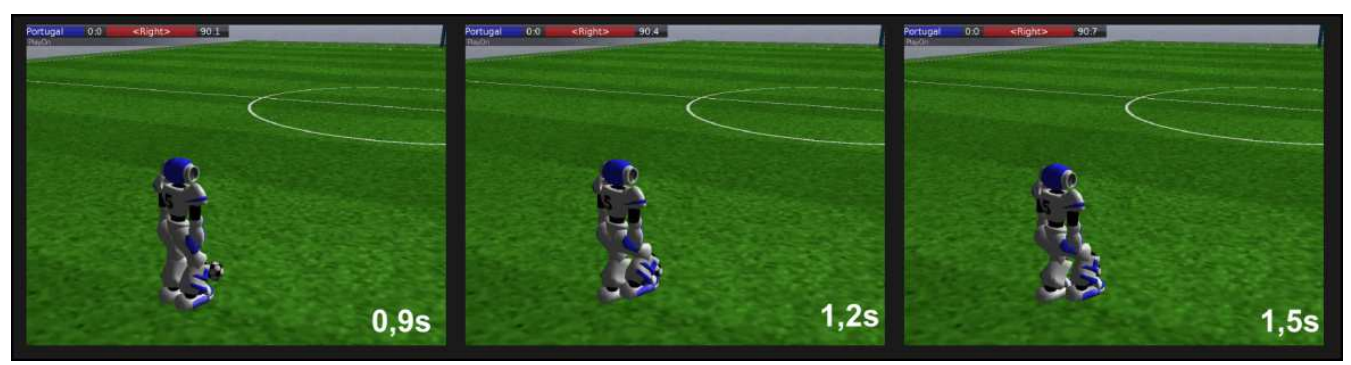

Figure 16. Sequence of the lowering and placing support foot

\subsubsection{Transfer of the robot's center of mass}

After lowering the robot it is necessary to transfer of center of mass of the robot from one foot to the other, to make possible the support of the robot's weight on the other foot. Since the position of the pelvis of the robot is kept within the working volume of both legs, it is possible to carry out a linear translation to the foot of same support required, as shown in Figure 17.

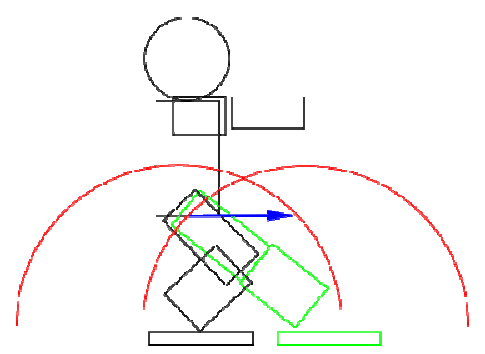

Figure 17. Transfer the robot's center of mass

The Figure 18 represents the center of mass transfer of the robot.

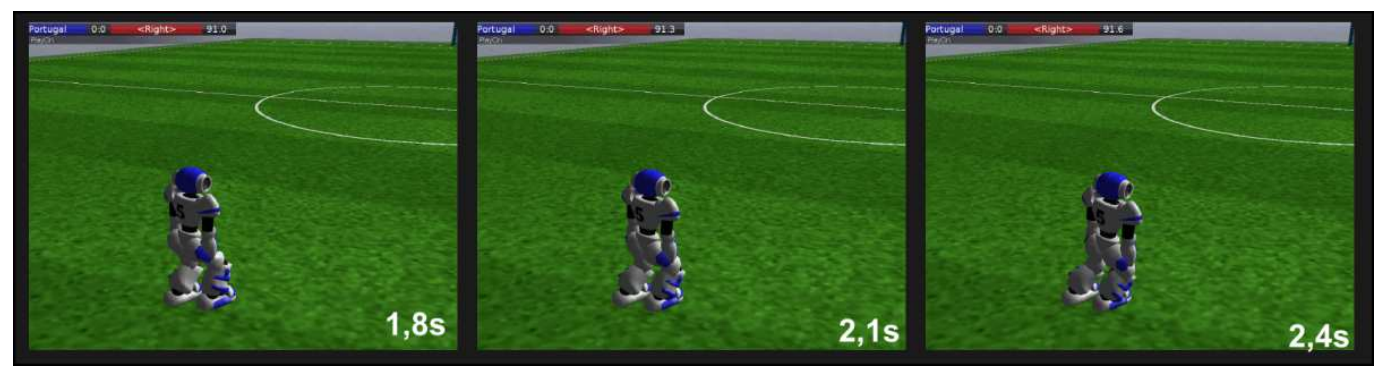

Figure 18. Transfer the robot's center of mass

\subsubsection{Kick's position}

After the center of mass transferred, the kick foot can be placed on the robot pose desired shot, as shown in Figure 19. Figure 20 represents several positions of the robot during kick's pose placing and kick. 


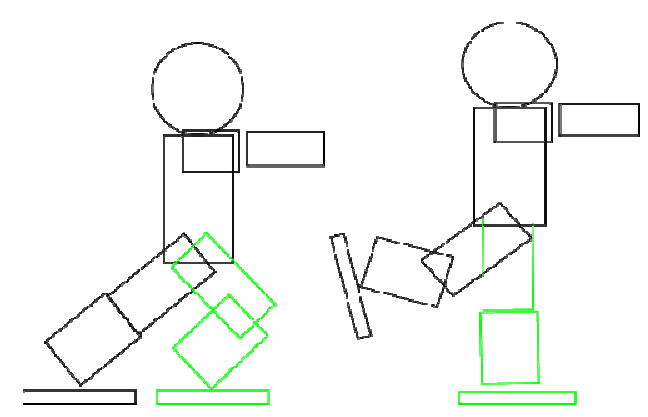

Figure 19. Pose after the final transfer of the center of mass of the robot pose and shot

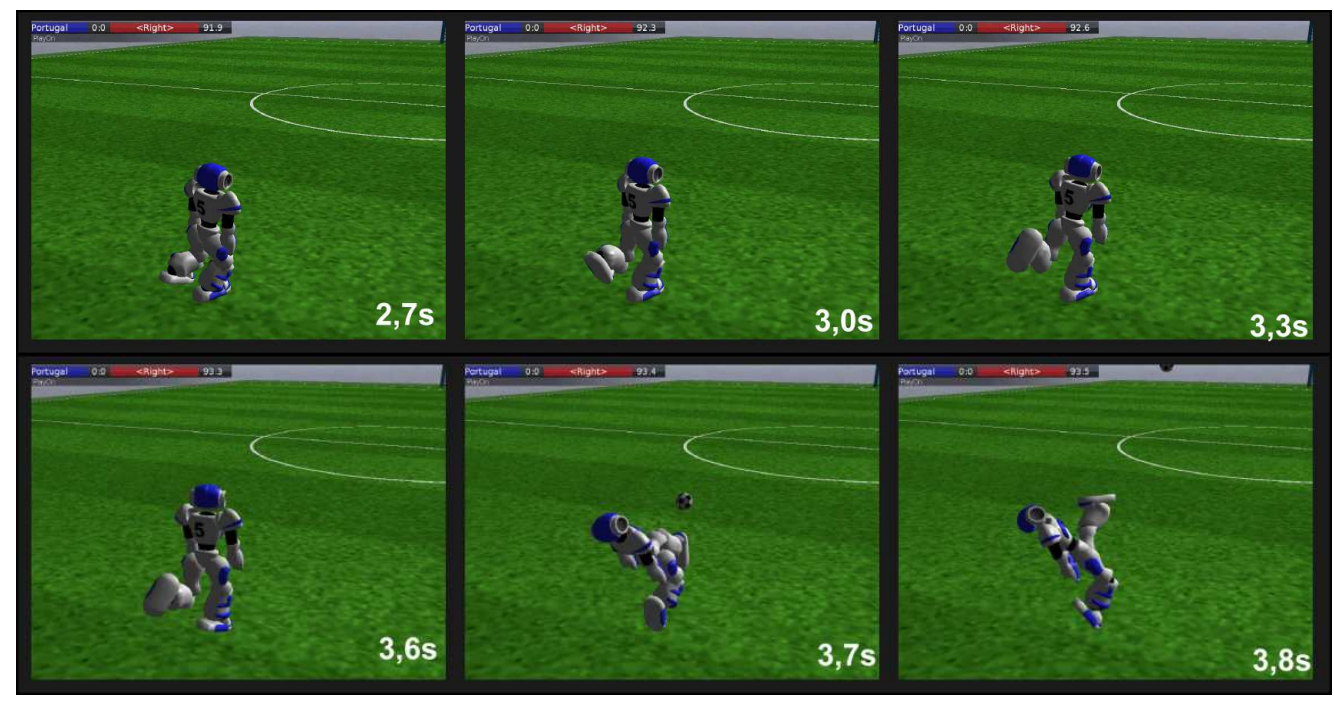

Figure 20. Sequence of the kick's poses placing and respective kick

\section{Tests and results}

The kick viable area was checked by performing several tests to the prepare kick movement. These tests consisted in positioning the robot in a set of predetermined positions and orientations relative to the ball and then verifying results in terms of distance and direction. Although the results obtained are subject to change, since the parameters defined in Section 3.1 can be changed through an optimization process, the ability to kick isn't as susceptible to these changes.

The area above the ball has been decomposed into a matrix with a resolution of $5 \mathrm{~cm}$, in which the robot is positioned. This decomposition was carried out for a variation of $x,(-0.25$ and 0$)$ meters, and $y,(-0.25$ and 0.25$)$ meters. At each point of the matrix, angles between $-50^{\circ}$ and $50^{\circ}$ with intervals of $10^{\circ}$ were tested and then two samples per test were made for results confirmation. To confirm the conditions that ensure the robot's ability to perform the prepare kick with his right foot, over 1300 tests were made and the results were analyzed with the MatLab tool. This analysis transformed the set of numbers into a gray scale image relating the distance traveled by the ball with the robot's initial pose.

The results after applying this transformation to all positions with the robot aligned with the target are shown in Figure 21. In this figure, the white color represents a null distance and the black color represents a distance travelled by the ball of 18 meters. The robot kick's performance is affected by the position where the movement was started.

Being defined the minimum distance as 7 meters to consider that the robot is capable of performing a shot, by applying a binarization to the referred image with the appropriate threshold, there was thus obtained the shot area provided by the ability to move to the right foot. To obtain the area of capacity to strike with both feet, a union was made between representative 
pictures of the results for a given orientation, with the mirror image of the representative results for the opposite orientation.

This result gave the following set of allowable shot areas according to the angle between the target and orientation of the player:

\subsection{Zero degree orientation}

Figure 22 represents the permissible area of shot in relation to the robot's position while it is at an angle of $0^{\circ}$ between its direction and the target. When the robot is aligned with the target, the allowable area is wide and has a set of very good results, with a maximum of 16.16 $\mathrm{m}$ recorded at position $(-25,5) \mathrm{cm}$. The two small areas of the shot recorded inadmissible at the bottom of the graph should overlap to the feet of the punch which makes it impossible to achieve. This approach is extremely favorable to the shot and has a vast area in which it is liable to be executed.

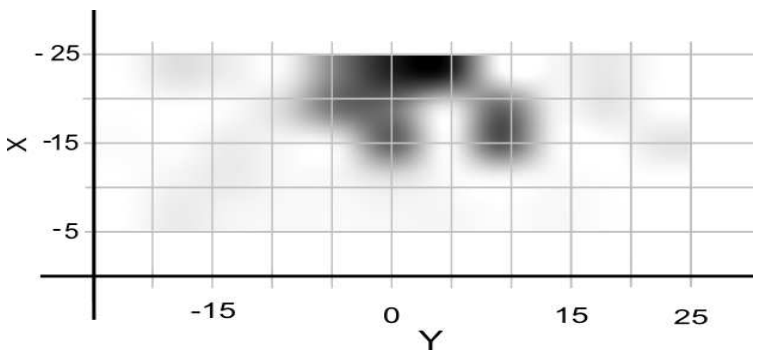

Figure 21. Representation of the kick distance relative to the robot's position when it is aligned with the target

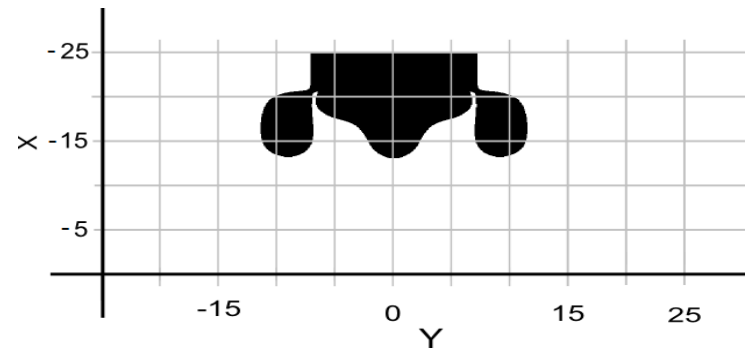

Figure 22. Representation of shooting allowable area from the position of the robot when it is at an angle of $0^{\circ}$ between their direction and the target

\subsection{Ten degree orientation}

Figure 23 represents the permissible area of shot in relation to the robot's position while it is at an angle of $10^{\circ}$ between the orientation an $d$ the target.

When the robot encounters an orientation of 10 degrees to the target, the permissible area remains extensive in many ways similar to the previous one. This orientation is very favorable to the shot and has a vast area in which it is liable to be executed. In this orientation, the preparation of shot has a set of very good results with a maximum of $16.69 \mathrm{~m}$ recorded at position $(-25,0) \mathrm{cm}$.

\subsection{Twenty degree orientation}

The Figure 24 represents the allowable shooting area in relation to the robot's position while it is at an angle of $20^{\circ}$ between the orientation and the target.

When the robot encounters an orientation of 20 degrees to the target, the admissible area is the largest, enabling a set of tests performed with more than $80 \mathrm{~cm}^{2}$. This approach is most favorable for the performance of the shot and has a set of excellent results with a maximum of $17.03 \mathrm{~m}$ recorded at position $(-20,5) \mathrm{cm}$.

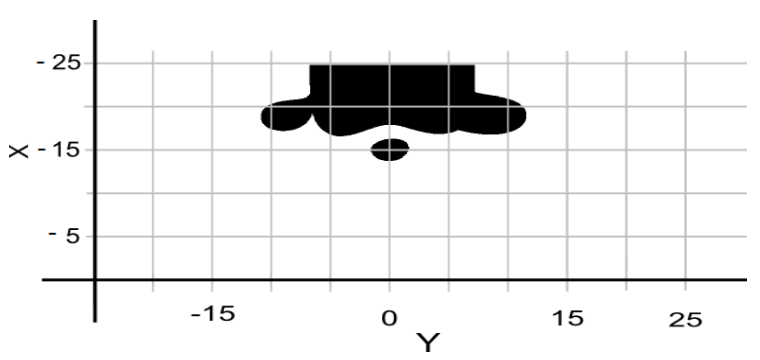

Figure 23. Representation of shooting allowable area from the position of the robot when it is at an angle of $10^{\circ}$ between the orientation and the target

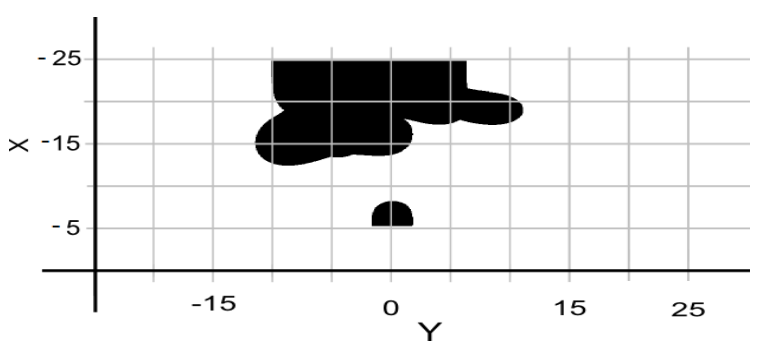

Figure 24. Representation of shooting allowable area from the position of the robot when it is at an angle of $20^{\circ}$ between the orientation and the target 


\subsection{Thirty degree orientation}

The Figure 25 represents the allowable shooting area in relation to the robot's position while it is at an angle of $30^{\circ}$ between the orientat ion and the target.

When the robot encounters an orientation of $30^{\circ}$ in relation to the target, the permissible area is slightly worse compared to previous results. Despite all, this approach has a number average results of up to $12.96 \mathrm{~m}$ recorded at position $(-20,0) \mathrm{cm}$.

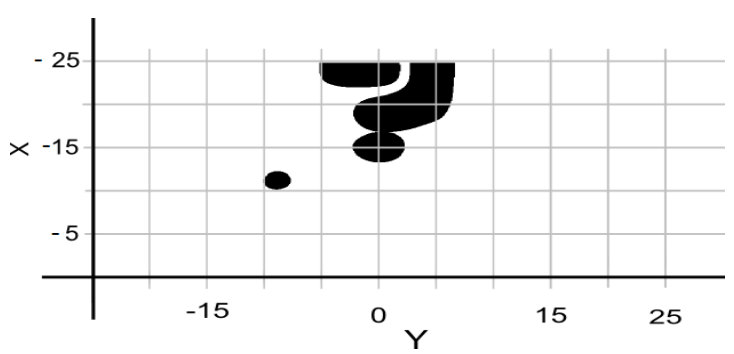

Figure 25. Representation of shooting allowable area from the position of the robot when it is at an angle of $30^{\circ}$ between the orientation and the target

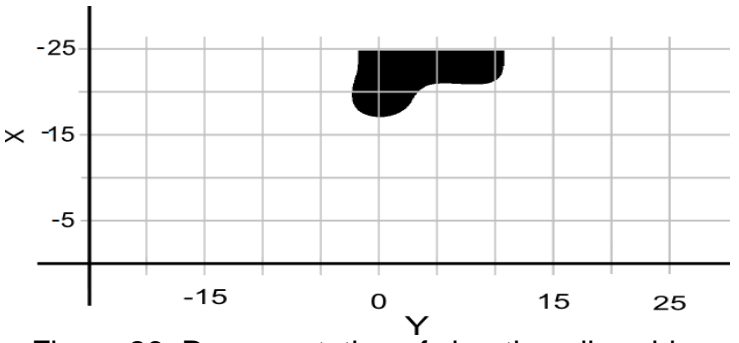

Figure 26. Representation of shooting allowable area from the position of the robot when it is at an angle of $40^{\circ}$ between the orientation and the target

\subsection{Forty degree orientation}

The Figure 26 represents the allowable shooting area in relation to the robot's position while it is at an angle of $40^{\circ}$ between the orientat ion and the target.

When the robot is in an orientation of $40^{\circ}$ in relat ion to its target, the acceptable area is a bit worse in comparison with previous results, being considerably reduced. Despite all, this approach has a number of results with a maximum of $12.74 \mathrm{~m}$ recorded at position $(-20,0) \mathrm{cm}$.

\subsection{Fifty degree orientation}

The Figure 27 represents the allowable shooting area in relation to the robot's position while it is at an angle of $50^{\circ}$ between the orientation and the target. When the robot encounters an orientation of $50^{\circ}$ in relation to the target, th e allowable area for shot preparation is greatly reduced. Nevertheless, given the extremely unfavorable conditions in orientation of the robot, this configuration has a set of acceptable results with a maximum of $13.06 \mathrm{~m}$ recorded at position $(-25,10) \mathrm{cm}$.

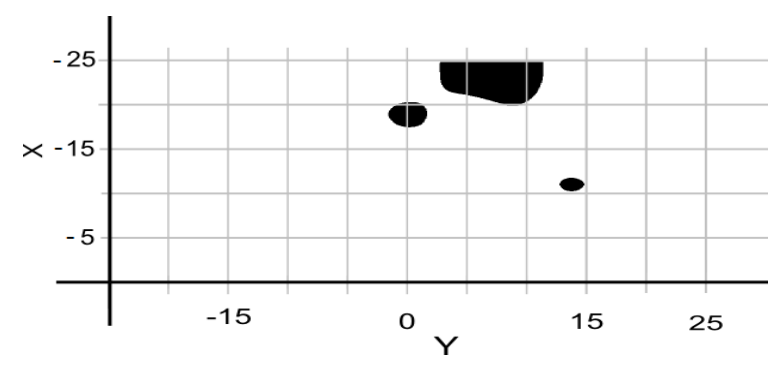

Figure 27. Representation of shooting allowable area from the position of the robot when it is at an angle of $50^{\circ}$ between the orientation and the target

\section{Conclusion}

In this work, a stability humanoid dynamic controller and a kick preparation movement were described. The stability controller allows the equilibrium evaluation, the control of the position of the center of mass, and the choice of the appropriate control method related to the response desired for the robot, constituting a vital tool for the control and safety of the robot and also a tool for evaluating and optimizing behavior.

Based on the stability module it was possible to develop a behavior for an omnidirectional kick preparation, providing a sequence for a single shot. An extensive set of tests proved the robustness and flexibility of the developed movement. 
The parameters used on these tests were hand tuned. Future work will consist in optimizing the parameters of the behavior of kick preparation for multiple sets of kicks, in developing a method of omnidirectional locomotion based on defined locomotion behavior, and incorporation of kick preparation on a stepping movement.

\section{Acknowledgements}

This work was partially funded by the ERDF - European Regional Development Fund through the COMPETE Programme (operational program for competitiveness) and by National Funds through FCT - Portuguese Foundation for Science and Technology within project "INTELLWHEELS - Intelligent Wheelchair with Flexible Multimodal Interface, RIPD/ADA/ 109636/2009".

\section{References}

[1] Hashimoto, S., Narita, S., Kasahara, H., et al. "Humanoid Robots in Waseda University-Hadaly-2 and WABIAN". Autonomous Robots. 2002; 12 (1): 25-38.

[2] Hirai, K., Hirose, M., Haikawa, Y., Takenaka, T. The Development of Honda Humanoid Robot. IEEE International Conference on Robotics \& Automation. Leuven, Belgium. 1993; 1321-1326.

[3] Aldebaran Robotics. NAO H25 - Humanoid Robot Platform Datasheet. 2011.

[4] Zhang, Z., He, D. Adaptive Tracking Control Algorithm for Picking Wheel Robot. TELKOMNIKA Indonesian Journal of Electrical Engineering. 2012; 10(8).

[5] Reis, L.P., Lau, N. "FC Portugal Team Description: RoboCup 2000 Simulation League Champion", RoboCup-2000: Robot Soccer World Cup IV, Springer LNAI, 2001; 2019: 29-40.

[6] Reis, L.P., Lau, N., Oliveira, E. "Situation Based Strategic Positioning for Coordinating a Team of Homogeneous Agents", Springer LNAI, 2001; 2103: 175-197.

[7] Mota, L., Reis, L.P., Lau, N. "Multi-Robot Coordination using Setplays in the Middle-size and Simulation Leagues". Mechatronics. 2011; 21(2): 434-444.

[8] Oliveira, J. Reis L.P., Faria, B.M. Empiric Evaluation of a Robot Dancing Framework based on MultiModal Events, TELKOMNIKA Indonesian Journal of Electrical Engineering. 2012; 10(8).

[9] Shafi, N., Reis, L.P., Lau, N. "Biped walking using coronal and sagittal movements based on the truncated Fourier series", Springer LNCS, LNAI 6556, 2011; pp. 324-335.

[10] Domingues, E., Lau, N., Pimentel, B., Shafi, N., Reis, L.P., Neves, A.J.R. "Humanoid behaviors: from simulation to a real robot", LNCS, LNAI 7026, 2011; pp. 352-364.

[11] Ziegler, J. G., Nichols, N. B. "Optimum Settings for Automatic Controllers". Journal of Dynamic Systems, Measurement, and Control. 1993; 115 (2B): 220-222. 